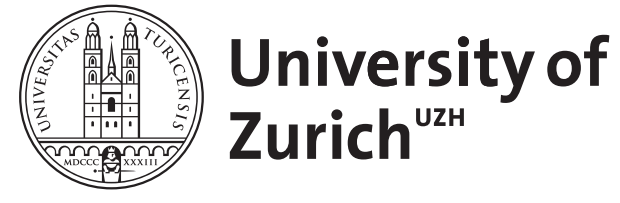

Zurich Open Repository and Archive

University of Zurich

University Library

Strickhofstrasse 39

CH-8057 Zurich

www.zora.uzh.ch

Year: 2021

\title{
Identifizierung von atypischem Asthma
}

Gaisl, Thomas

DOI: https://doi.org/10.1024/1661-8157/a003743

Posted at the Zurich Open Repository and Archive, University of Zurich

ZORA URL: https://doi.org/10.5167/uzh-211980

Journal Article

Accepted Version

Originally published at:

Gaisl, Thomas (2021). Identifizierung von atypischem Asthma. Praxis, 110(14):767.

DOI: https://doi.org/10.1024/1661-8157/a003743 


\section{Editorial}

Thomas Gais| ${ }^{1,2}$

${ }^{1}$ Klinik für Pneumologie, Universitätsspital Zürich, Zürich, Schweiz

${ }^{2}$ Harvard T.H. Chan School of Public Health, Harvard University, Boston MA, USA

Asthma bronchiale ist eine der häufigsten respiratorischen Erkrankungen weltweit und ist bis heute medikamentös nicht komplett kontrollierbar. Mit einer Prävalenz von ca. 5\% in der Bevölkerung, werden jährlich 420'000 Todesfälle registriert. ${ }^{1}$

\section{Ein buntes Bild}

Von einem klinischen Standpunkt aus betrachtet, ist die Gruppe der Patienten mit Asthma bronchiale auffallend heterogen. ${ }^{2}$ Im Gegensatz zu vielen anderen internistischen Erkrankungen, ist u.a. jedes Alter bei dieser chronischen Erkrankung vertreten und der klinische Verlauf ist oft unvorhersehbar. Der Zeitpunkt der Erstmanifestation reicht oft vom Vorschulalter bis hin zur Pension, das komplette Spektrum der allergischen Diathese ist abgebildet, einzelne Ko-Faktoren wie z.B. Rauchen und die gastroösophageale Refluxkrankheit sind unterschiedlich stark ausgeprägt und die Asthma-spezifischen Therapien sind unterschiedlich effektiv.

Basierend auf ersten empirischen Untersuchungen, wurden Begriffe wie «intrinisches Asthma», "Milbenasthma» oder "morning-dipping Asthma» eingeführt. In der Literatur und in den Lehrbüchern wurden verschiedene Konzepte für die Phänotypisierung verwendet und man tat sich nicht immer leicht, die Krankheit von der chronisch obstruktiven Lungenkrankheit (COPD) abzugrenzen. Man realisierte zunehmend, dass "Asthma bronchiale» ein Sammelbegriff für verschiedene respiratorische Erkrankungen mit teilweise unterschiedlichen molekularen Mechanismen ist.

\section{Identifizierung des Typ-2 Asthma}

Im aktuellen Mini-Review von Thomas Rothe und Tsogyal Daniela Latshang ${ }^{3}$ werden die seit den 1970er-Jahren gewonnenen Erkenntnisse zusammengefasst und die Herauskristallisierung der sogenannten «Typ-2-Asthma Phänotypen» beschrieben, welche sich durch ihre atypische Manifestation kennzeichnen. Mit anderen Worten, diese Phänotypen charakterisieren sich durch ihre "Diskonkordanz» zwischen den klinischen Symptomen und eosinophiler Inflammation.

Obwohl der genaue Grund für diese Diskonkordanz weiterhin nicht genau verstanden ist, macht es diese Gruppe an Patienten nicht weniger interessant. Im Gegenteil, der Einzug von subkutan verabreichbaren Biopharmazeutika (sog. „Biologicals") erweiterte neben den Therapieoptionen auch das pathophysiologsiche Verständnis für den Sammelbegriff „Asthma bronchiale".

Mit heutigem Stand, sind in der Schweiz mit Reslizumab, Benralizumab, Mepolizumab (AntiIL-5), Omalizumab (Anti-IgE) und Dupilumab (anti-IL-4, anti-IL-13) fünf humanisierte monoklonale Antikörper als Antiasthmatikum zugelassen. Verschiedene Aspekte der Asthma- 
Phänotypen finden sich auch in den Indikationsvoraussetzungen für die Biologicals wieder, was die Wichtigkeit der aktuellen Asthma-Phänotypen für die klinische Praxis unterstreicht.

Das aktuelle Mini-Review beschreibt, wie ebendiese atypischen Patienten mit Asthma bronchiale anhand von klinischen, laboranalytischen und lungenfunktioneller Dynamik identifiziert werden können und wo insbesondere eine Verwechslungsgefahr mit anderen Erkrankungen besteht. Aufgrund der heute verfügbaren Therapieoptionen, lohnt sich diese Reise, wenn auch noch nicht das letzte Wort geschrieben wurden.

\section{Referenzen}

1. Naghavi $\mathrm{M}$ et al. Global, regional, and national age-sex specific mortality for 264 causes of death, 1980-2016: A systematic analysis for the Global Burden of Disease Study 2016. Lancet. 2017;390(10100):1151-210.

2. L-P. Boulet. Influence of comorbid conditions on asthma. European Respiratory Journal 2009 33: 897-906

3. Thomas Rothe und Tsogyal Daniela Latshang. Typ-2 Asthma: Leaving behind the Perspective of the Seventies. Praxis. ... 